\title{
Serratia marcescens Discospondylitis in a White Stork (Ciconia ciconia)
}

\author{
Patrícia Poeta $^{1,2 *}$, Roberto F. Sargo ${ }^{3}$, Joana M. Valente ${ }^{3}$, Justina Prada-Oliveira ${ }^{1,4}$, Filipe C. Silva ${ }^{1}$, \\ Célia M.F. Felício ${ }^{5}$, Luís M. Costa ${ }^{1,4}$, Joana Teixeira ${ }^{1}$, José E. Pereira ${ }^{1,4}$ and Artur S.P. Varejão ${ }^{1,4}$ \\ ${ }^{1}$ Department of Veterinary Sciences, University of Trás-os-Montes and Alto Douro (UTAD), 5001-801, Vila Real, Portugal \\ ${ }^{2}$ UCIBIO-REQUIMTE, Chemistry Department, Faculty of Science and Technology, University NOVA of Lisbon, 2829-516, Caparica, Lisbon, Portugal \\ ${ }^{3}$ Veterinary Medical Teaching Hospital of University of Trás-os-Montes e Alto Douro, 5001-801 Vila Real, Portugal \\ ${ }^{4}$ Veterinary and Animal Science Research Center (CECAV) \\ ${ }^{5}$ Service of Imagiology, Hospital Center of Trás-os-Montes e Alto Douro, E.P.E., Vila Real, Portugal
}

Received: August 22, 2016; Accepted: September 19, 2016; Published: September 23, 2016

*Corresponding author: Patrícia Poeta, Department of Veterinary Sciences, University of Trás-os-Montes and Alto Douro (UTAD), 5001-801, Vila Real, Portugal, Tel.: +351 259350 466; fax: +351 259350 629; E-mail: ppoeta@utad.pt

\begin{abstract}
An adult white stork (Ciconia ciconia) was admitted to the Wild Animal Rehabilitation Centre of the Veterinary Medical Teaching Hospital at the University of Trás-os-Montes e Alto Douro for examination after a road traffic accident. A fracture of the first caudal vertebra was diagnosed based on the radiological findings. 4 weeks after admission a strange postural stance was noticed along with hyporexia. Radiography revealed lytic lesions on the intervertebral spaces of the caudal thoracic vertebra, that weren't present in the previous exam. Based on the clinical, hematological and radiological findings a presumptive diagnosis of discospondylitis was established. A computed tomography of the spine revealed large lytic lesions and proliferation of granulomatous tissue affecting the vertebral ends of the caudal thoracic vertebras. The animal was humanly euthanized and immediately after death, sterile samples of intracardiac blood and from the lytic lesions were obtained and cultured. All samples from the lytic lesions showed growth of pure cultures of Serratia marcescens. To the authors' knowledge this is the first report of discospondylitis caused by these bacteria in birds, as seen in humans.
\end{abstract}

Keywords: Avian; Ciconia ciconia; Computed tomography; Diagnostic imaging; Discospondylitis; Serratia marcescens, White stork

\section{Abbreviation}

CBC: Complete Blood Cell Count; CT: Computed Tomography

\section{Introduction}

The Serratia marcescens is a gram-negative bacillus that belongs to the Enterobacteriaceae family [1,2]. These ubiquitous bacteria can be found mainly in water, plants and soil [3].

In humans it causes opportunistic and nosocomial infections and it has been isolated from several infections such as septicemia, meningitis, conjunctivitis, cystitis, osteomyelitis, respiratory and urinary tract infections, among others $[2,3]$.

Several infections by Serratia were reported on animals such as mastitis in dairy cows, endocarditis and sepsis in horses, sepsis and osteomyelitis in dogs and cats, intracranial abscesses and/or purulent meningoencephalitis in deer, pneumonia in sea lions, and localized articular and cutaneous infections in reptiles. It was also reported a case enteritis in a bird (Swallow-tailed Hummingbird) by Serratia marcescens [2]. The clinical signs of birds infected with Serratia marcescens include vomiting, hepatitis, nephritis, and septicemia and usually leads to death [2].

Although there are reported cases of bird infections and death with Serratia marcescens, this may not happen to all species since there are also reports of isolation of these bacteria in apparently healthy birds. In fact, some species may be carriers for these bacteria and could be the source of zoonotic infections [2].

Here we analyze another Serratia marcescens bird infection that leads to the death of a wild white stork.

\section{Patients and Methods}

A wild adult white stork (Ciconia ciconia) was admitted in the Wild Animal Rehabilitation Centre of the Veterinary Medical Teaching Hospital at the University of Trás-os-Montes e Alto Douro, several hours after a road traffic accident.

It was made a physical and a neurological exam after the admittance. A blood sample was submitted for a CBC, plasma biochemical profile and plasma protein electrophoresis, and whole body radiographs were taken. The animal was admitted to the quarantine with a plan of complete rest in cage for 15 days and adjunctive therapy with carprofen $4 \mathrm{mg} / \mathrm{kg}(8,8 \mathrm{mg} / \mathrm{lb})$ once daily in forced food for 7 days. An IV catheter was placed for fluid therapy and vitamin Bb support until the stork started eating by itself.

New whole body radiographs were taken and a new neurologic exam was made. It was also necessary to submit a new blood sample for a CBC, plasma biochemical profile and plasma protein electrophoresis. At this time the animal was on antibiotic therapy (enrofloxacin $15 \mathrm{mg} / \mathrm{kg}$ [33 mg/ lb] PO q 12h) 
for the treatment of diarrhea, after a stool smear demonstrated prevalence of gram negative bacteria. Itraconazole $10 \mathrm{mg} / \mathrm{kg}(22$ $\mathrm{mg} / \mathrm{lb}) \mathrm{q} 12 \mathrm{~h}$ and carprofen, $4 \mathrm{mg} / \mathrm{kg}(8,8 \mathrm{mg} / \mathrm{lb}) \mathrm{q} 24 \mathrm{~h}$, were added to the regime and the animal returned to the quarantine for continuous monitoring. As the stork was already on antibiotic therapy, no blood culture was performed.

Another neurological exam was made. Under general anesthesia (propofol $6 \mathrm{mg} / \mathrm{kg}(13,2 \mathrm{mg} / \mathrm{lb}) \mathrm{IV}$ ad efectum) a CT was performed with a HI Speed NX/I CT scanner (GE Medical Systems, Pewaukee, Wisconsin, USA) to further investigate the affected spine.

The stork was humanly euthanized and after euthanasia the animal was plucked and the skin was aseptically prepared. The coelomic cavity was incised and the affected intervertebral spaces identified. Sterile samples from the affected intervertebral spaces were collected and samples of, intracardiac blood and peripheral tissues (lung, air sac and liver) were also retrieved and packed in sterile containers for microbiologic and fungal cultures. Necropsy proceeded following protocol. The caudal thoracic and abdominal air sacs where thickened and the caudal thoracic air sac was adhered to the liver capsule. The vertebral column was transected at the 1st thoracic vertebra level and at the end of the first fused synsacrum vertebra.

The thoracic vertebrae were transected laterally to expose the medullary cavity and all thoracic vertebrae were then fixed in formaldehyde along with fragments of the internal organs for histopathology.

\section{Results}

The initial physical exam revealed a mild dehydration (6\%), increased respiratory sounds from both lungs, a crepitation was noted on the caudal part of the synsacrum and also a ventral deviation of the pygostyle. The pygostyle and the tail feathers were fixed in an over flexed position. The first neurological exam revealed no proprioceptive or sensory deficits in the rear limbs; the vent sphincter reflex was absent. The stork had an overdistended and easily expressed cloaca. The lesion was localized to the pudendal plexus. No abnormalities were observed on the CBC and plasma biochemical profile or plasma protein electrophoresis first analysis. A fracture of the first caudal vertebra was diagnosed without any other spinal lesions.

Four weeks after admittance a period of hyporexia was noticed, along with a strange postural stance. The stork spent most of the time standing on both feet; with a hunched posture, more prominent at the transition between the free thoracic vertebrae and the synsacrum. Lytic lesions on the intervertebral spaces of the last three free thoracic vertebrae were noticed after taken the new whole body radiographs (Figure 1). The neurologic exam revealed no proprioceptive or sensory deficits in the rear limbs and no pain was present on palpation of the spine. The CBC revealed a leukocytosis (35400 cells/ $\mu \mathrm{L}$; reference interval, $17400-25800$ cells/ $\mu \mathrm{L}$ ) with heterophilia (23718 cells / $\mu \mathrm{L}$; reference interval, 9900-16300 cells/ $\mu \mathrm{L}$ ), associated with hypoalbuminemia (0.7 g/ dL; reference interval, 1.1-1.6 g/
$\mathrm{dL})$ and an increase in $\alpha 1(1.06 \mathrm{~g} / \mathrm{dL}$; reference interval, 0.2$0.8 \mathrm{~g} / \mathrm{dL}$ ) and $\alpha 2$ (1.14 g/ dL; reference interval, 0.1-0.5 g/ dL) globulins. Based on the clinical, hematological and radiologic findings a presumptive diagnosis of acute discospondylitis was established.

On the following days the stork started eating normally but the postural stance kept abnormal. If left undisturbed, the stork would not move and would remain lying on the keel. Two weeks later the follow up radiographs showed no improvement of the lytic lesions and the pulmonary field, surrounding the lesions, had an increased homogenous density. After the new neurological exam pain was perceived when digital compression on the free thoracic vertebrae was applied.

Transverse CT images and multiplanar reconstructions showed sclerotic endplates and multiple, irregular regions of bony lysis in the endplates of the affected thoracic vertebrae and proliferation of granulomatous tissue, consistent with discospondylitis (Figure 2A and 2B). As this stork couldn't be released back into the wild and no improvement of the discospondylitis was noticed (pain signs were still present), the stork was humanly euthanized.

After euthanasia granulomatous lesions were observed on the intervertebral spaces of the last three free thoracic vertebrae (Figure 3) and no compression of the spinal cord was evident. No other remarkable abnormalities were found.

The microbiological culture revealed a pure culture of Serratia marcescens in all tissues sampled and a negative culture from the intracardiac blood. No fungal growth was detected in all samples. This strain showed resistance to amoxicillin, amoxicillin with clavulanic acid, tetracycline, cefradine, enrofloxacin, chloramphenicol, and susceptibility to gentamicin, cefoperazone, neomycin and trimethoprim with sulfadimethoxine.

Histopathologic examination of the sampled tissues revealed necrotic and granulomatous lesions of bone, with abscessed areas in the articular ends of the vertebral bodies. The inflammatory infiltrate was extensive with heterophils, lymphocytes, histiocytes, osteoclasts, plasma cells, and multinucleated giant cells. Granulation tissue was also observed. A large number of gram negative bacteria are seen in the necrotic debris. The

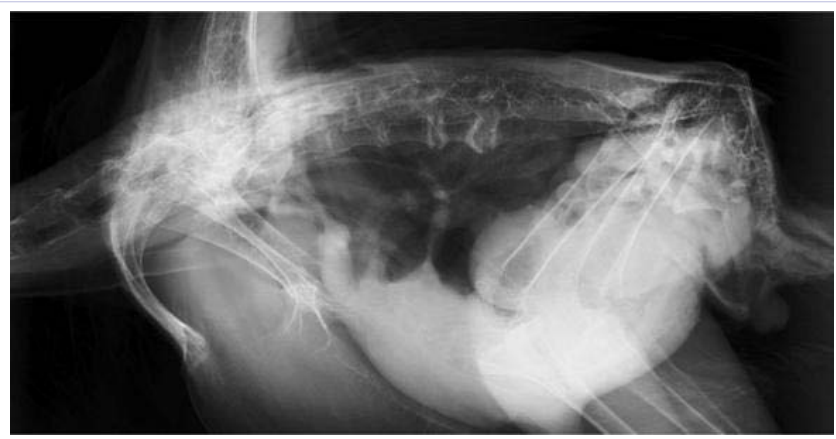

Figure 1: Lateral radiograph of the white stork at the time of diagnosis. Note the lytic appearance on the intervertebral spaces of the last three free thoracic vertebrae, characteristic of discospondylitis. 
lung presented antracosis, and the liver presented a discrete lymphocytic infiltrate on the portal areas. The remaining organs were not affected.

\section{Discussion}

A case of discospondylitis was diagnosed in a Ciconia ciconia based on the clinical history and on the results of hematologic testing, radiography and CT imaging. Bacteriological culture of the affected intervertebral disc rendered a pure culture of Serratia marcescens, an important nosocomial pathogen $[1,2,4]$ To the authors' knowledge, there have been no previous reported cases of discospondylitis in Ciconiiformes; moreover, spinal diseases are not documented in the reviewed literature for this avian group.

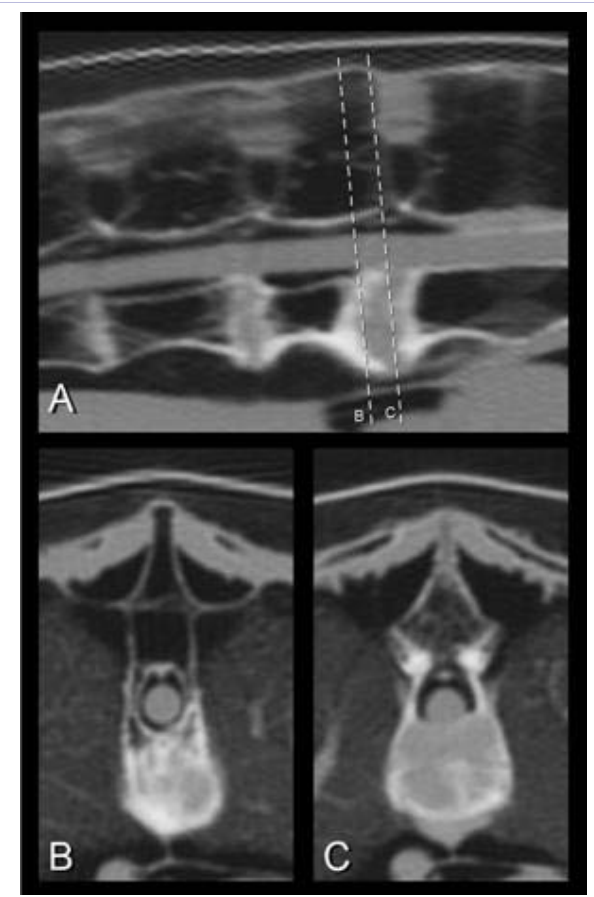

Figure 2: Computed Tomography (CT) of the white stork at the time of diagnosis, bone window. (a) Reformatted sagittal CT scan makes visualization of the lytic areas more evident and reveals proliferation of granulomatous tissue not previously identified. The dotted lines, at the level of the disk space between the last free thoracic vertebra and the synsacrum represents the transverse planes in (b) and (c).
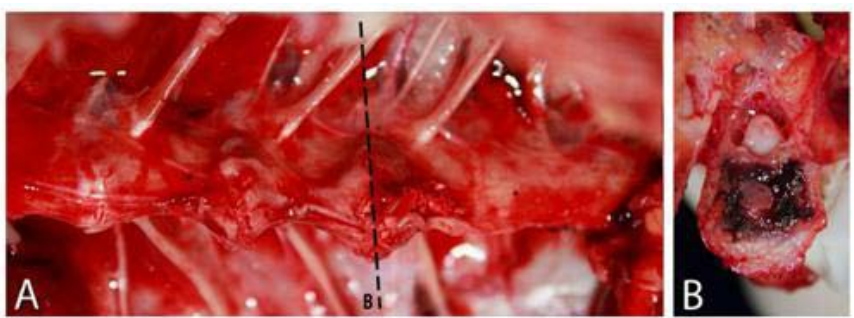

Figure 3: (a) Ventral view of the 3 disk spaces affected on gross necropsy, showing the granulomatous lesions. The dotted line, at the level of the disk space between the last free thoracic vertebra and the synsacrum represents the transverse plane observed in (b), revealing a haemorrhagic lesion, with a central pale area resembling a granuloma.
Discospondylitis is an infectious, inflammatory disease centered on the intervertebral disc and involving the vertebral endplates and adjacent bone of the vertebral body [5]. There are only two reports of avian discospondylitis, in a free-ranging yellow-eyed penguin (Megadyptes antipodes) [6] and in a blackedfooted penguin (Spheniscus demersus) [7].

This inflammatory condition is a common cause of spinal hyperaesthesia in dogs, where associated neurologic dysfunction may result from proliferation of fibrous tissue and bone in response to inflammation and instability, collapse of the intervertebral space and/or pathological vertebral fractures. Approximately $30 \%$ of affected dogs have signs of systemic illness such as anorexia, fever and weight loss.7 In this case only pain, hyporexia and depression associated to a strange postural stance were recognized. Early case reports in two penguins revealed that both pain and lumbosacral kyphosis were present, and associated neurologic signs affected one of them [6,7].

Traditionally, radiography has been used to support the diagnosis of discospondylitis in veterinary medicine, [8] although Field, et al. [7] did not find any radiographic alteration in their case. However, it should be noted that radiographic changes may not be evident in the first weeks of infection [8]. In this stork lytic lesions were noticed 4 weeks after the trauma. The radiographic findings of our case included erosion of the endplates and a widened disk spaces due to the lysis of the adjacent bone. CT is a tomographic modality that identifies subtle endplate erosion, provides more detailed images and can detect inflammation of the paravertebral tissues. It is also useful in monitoring the progression of the disease [6,9]. In this clinical case sclerotic endplates and destruction of the vertebrae bodies were clearly visualized.

The precise pathophysiology of discospondylitis is unclear. The source of hematogenous cause of discospondylitis in animals often remains undetermined but may rise from urogenital, skin, respiratory and oral infections [10]. The reduced velocity of blood supply associated to the porous nature of the endplates may provide a route into the nucleus pulposus for bacterial and fungal organisms [8]. In a case series of 513 dogs with discospondylitis traumatic injury with transient bacteremia has also been implicated as an initiating factor [10]. In this case, the stork suffered a road traffic accident with spinal complications. The fracture on the 1st caudal vertebra with the desensitized pygostyle and its ventral over flexed position did not allow a good flight control. The absence of the vent sphincter reflex and the overdistended cloaca that was easily expressed indicate lower motor neuron damage to the pudendal nerve or its roots [11-13] and no improvement of this condition was noticed overtime.

The incidence of traumatic and infectious lesions in the vertebral column of birds seems to be higher in the free thoracic vertebrae, as their articulation to the stiff synsacrum is subject to mechanical stress [6,14-17]. In this case the more prominent lesion was also localized in the intervertebral space between the last free thoracic vertebra and the synsacrum. Trauma after collision, with hematoma formation and posterior hematogenous 
bacterial colonization is supposed to be the pathologic mechanism of similar lesions on hunting raptors [17].

Percutaneous needle aspiration or open biopsy from the disc space is more sensitive for identification of the causative agent than blood or urine cultures. These procedures have yielded positive culture in approximately $75 \%$ of dogs [18]. Staphylococcus spp. are the most frequent isolated bacteria in humans [19], dogs and poultry affected with discospondylitis $[6,7]$. Nevertheless other microorganisms have been cultured, including fungus [6]. In the free-rangingyellow-eyed penguin case no bacteria was isolated, but a Corynebacterium amycolatum was suspected [6] and in the blacked-footed penguin Staphylococcus aureus was cultured [7]. Although no skin lesions were detected in our bird, the cloaca was always distended and diarrhea was present, with gram-negative bacteria overgrowth.

Culture of the affected intervertebral disc rendered a pure culture of Serratia marcescens. On Lau, et al. [19] review, only 11 cases of Serratia marcescens vertebral infection were reported between 1976 and 2012, in human patients. Serratia marcescens is a ubiquitous gram negative bacillus of the Enterobacteriaceae family. It has been associated with nosocomial infection on children and immunosuppressed human patients [1,2,20]. This bacterium has been isolated from mastitis, sepsis, endocarditis, osteomyelitis, necrotizing fasciitis and dermal ulcers, intracranial abscesses and purulent meningoencephalitis, and pneumonia of a varied range of mammal species [2,21]. In reptiles, localized articular and dermal ulcers were associated with Serratia marcescens cultures [2]. Del-Pozo, et al. [4] recently described a necrotizing myocarditis in a Mexican axolotl (Ambystoma mexicanum). Serratia marcescens is seldom isolated from avian species [2]. Although associated with chronic debilitating disease in psittacine birds, Serratia marcescens strains have been isolated from droppings of healthy birds including Houbara bustards (Chlamydotis undulata), Raptors and Passeriformes [2]. In birds, bone or articular lesions due to Serratia marcescens infections have not been described, until this report. Serratia marcescens usually shows sensitivity to third-generation cephalosporins, and equal quantities of imipenem and cilastatin. Serratia spp. have been found to be resistant to ampicillin, amoxicillin, penicillin $\mathrm{G}$, tetracyclines, cefazolin, rifampin, lincosamides, glycopeptides and others $[3,22]$.

Although few reports exist of infectious spinal diseases in avian species, discospondylitis should be part of the differentials for spinal pain, kyphosis, fever, motor dysfunction, anorexia and depression. Moreover, Serratia marcescens must be taken into account as possible pathogen in ill wild birds that inhabit and feed in marsh habitats.

\section{Acknowledgements}

The authors would like to acknowledge, Rosa Barbosa for English language revision.

\section{Footnotes}

a - Rimadyl, Pfizer Inc., New York, USA

b - Duphalyte, Pfizer Inc., New York, USA c - Baytril, Bayer Healthcare, Kiel, Germany

d - Sporanox, Janssen Pharmaceuticals, New Jersey, USA

e - Lipuro 1\%, B. Braun, Melsungen, Germany

\section{Compliance of Ethical Standards}

\section{Conflict of interest}

All authors declare that they have no conflicts of interest. No author has a commercial or other association that might pose a conflict of interest (e.g., pharmaceutical stock ownership, consultancy, advisory board membership, relevant patents, or research funding).

\section{References}

1. Hejazi A, Falkiner FR. Serratia marcescens. J Med Microbiol. 1997;46(11):903-12.

2. Saidenberg AB, Teixeira RH, Astolfi-Ferreira CS, Knöbl T, Ferreira AJ. Serratia marcescens infection in a swallow-tailed hummingbird. J Wildl Dis. 2007;43(1):107-10.

3. Fusté E, Galisteo GJ, Jover L, Vinuesa T, Villa TG, Viñas M. Comparison of antibiotic susceptibility of old and current Serratia. Future Microbiol. 2012;7(6):781-6. doi: 10.2217/fmb.12.40.

4. Del-Pozo J, Girling S, Pizzi R, Mancinelli E, Else RW. Severe Necrotizing Myocarditis caused by Serratia marcescens Infection in an Axolotl (Ambystoma mexicanum). J Comp Pathol. 2011;144(4):334-8. doi: 10.1016/j.jcpa.2010.11.001.

5. Kornegay JN, Barber DL. Diskospondylitis in dogs. J Am Vet Med Assoc. 1980;177(4):337-41.

6. Bergen DJ, Gartrell BD. Discospondylitis in a yellow-eyed penguin (Megadyptes antipodes). J Avian Med Surg. 2010;24(1):58-63.

7. Field CL, Beaufrère H, Wakamatsu N, Rademacher N, MacLean R. Discospondylitis caused by Staphylococcus aureus in an African black-footed penguin (Spheniscus demersus). J Avian Med Surg. 2012;26(4):232-8.

8. Thomas WB. Diskospondylitis and other vertebral infections. Vet Clin North Am Small Anim Pract. 2000;30(1):169-82, vii.

9. Gonzalo-Orden JM, Altónaga JR, Orden MA, Gonzalo JM. Magnetic resonance, computed tomographic and radiologic findings in a dog with discospondylitis. Vet Radiol Ultrasound. 2000;41(2):142-4.

10. Burkert BA, Kerwin SC, Hosgood GL, Pechman RD, Fontenelle JP. Signalment and clinical features of diskospondylitis in dogs: 513 cases (1980-2001). J Am Vet Med Assoc. 2005;227(2):268-75.

11. Clippinger TL, Bennett RA, Platt SR. The Avian Neurologic Examination and Ancillary Neurodiagnostic Techniques: A Review Update. Vet Clin North Am Exot Anim Pract. 2007;10(3):803-36, vi.

12. Jones MP, Orosz SE. Overview of avian neurology and neurological diseases. Seminars in Avian and Exotic Pet Medicine. 1996;5(3):150164.

13. Platt SR. Evaluating and treating the nervous system. In: Harrison GJ, Lightfoot TL, eds. Clinical Avian Medicine. Vol II. Palm Beach, FL: Spix Publishing. 2006;493-517.

14. Martin LT, Martin MP, Barnes HJ. Experimental reproduction of enterococcal spondylitis in male broiler breeder chickens. Avian Dis. 2011;55(2):273-8. 
15. Bennett RA. Neurology. In: Ritchie BW, Harrison GJ, Harrison LR, eds. Avian Medicine: Principles and Application. Lake Worth, FL: Wingers Publishing. 1994;723-747.

16. Harcourt-Brown NH. Chapter 3: Orthopedic conditions. In: Birds of Prey: Anatomy, Radiography and Clinical Conditions of the Pelvic Limb. Lake Worth, FL : Zoological Education Network; 2000;220.

17. Stalker MJ, Brash ML, Weisz A, Ouckama RM, Slavic D. Arthritis and osteomyelitis associated with Enterococcus cecorum infection in broiler and broiler breeder chickens in Ontario, Canada. J Vet Diagn Invest. 2010;22(4):643-5.

18. Platt S, Freeman C. In: Platt S, Olby N. eds. BSAVA Manual of Canine and Feline Neurology. 4th ed. Gloucester, England: BSAVA. 2013;252270.
19. Lau JX, Li JY, Yong TY. Non-contiguous multifocal vertebral osteomyelitis caused by Serratia marcescens. Mod Rheumatol. 2015;25(2):303-6. doi: 10.3109/14397595.2013.874754.

20. Voelz A, Müller A, Gillen J, Le C, Dresbach T, Engelhart S, et al. Outbreaks of Serratia marcescens in neonatal and pediatric intensive care units: Clinical aspects, risk factors and management. Int J Hyg Environ Health. 2010;213(2):79-87. doi: 10.1016/j.ijheh.2009.09.003.

21. Perez C, Fujii Y, Fauls M, Hummel J, Breitschwerdt E. Fatal aortic endocarditis associated with community-acquired Serratia marcescens infection in a dog. J Am Anim Hosp Assoc. 2011;47(2):1337. doi: 10.5326/JAAHA-MS-5616.

22. Peddle G, Sleeper MM. Canine bacterial endocarditis: a review. J Am Anim Hosp Assoc. 2007;43(5):258-63. 